\title{
Automatic Date Fruit Recognition Using Outlier Detection Techniques and Gaussian Mixture Models
}

\author{
Oussama Aiadi ${ }^{*}$, Mohammed Lamine Kherfi ${ }^{+,}$, Belal Khaldi* \\ * University of Kasdi Marbah, LAGE Laboratory, Ouargla, Algeria. \\ ${ }^{+}$Université du Québec à Trois-Rivières, LAMIA Laboratory, Trois-Rivières, Canada
}

Received 29th of August 2017; accepted 30 ${ }^{\text {th }}$ march 2019

\begin{abstract}
In this paper, we propose a method for automatically recognizing different date varieties. The presence of outlier samples could significantly degrade the recognition outcomes. Therefore, we separately prune samples of each variety from outliers using the Pruning Local Distance-based Outlier Factor (PLDOF) method. Samples of the same variety could have several visual appearances because of the noticeable variation in terms of their visual characteristics. Thus, in order to take this intra-variation into account, we model each variety with a Gaussian Mixture Model (GMM), where each component within the GMM corresponds to one visual appearance. Expectation-Maximization (EM) algorithm was used for parameters estimation and Davies-Bouldin index was used to automatically and precisely estimate the number of components (i.e., appearances). Compared to the related studies, the proposed method 1) is capable to recognize samples though the noticeable variation, in terms of maturity stage and hardness degree, within some varieties; 2) achieves a high recognition rate in spite of the presence of outlier samples; 3 ) is capable to distinguish between the highly confusing varieties; 4) is fully automatic, as it does not require neither physical measurements nor human assistance. For testing purposes, we introduce a new benchmark which includes the highest number of varieties (11) compared to the previous studies. Experiments show that our method has significantly outperformed several methods, where a high recognition rate of $98.65 \%$ has been reached.
\end{abstract}

Key Words: date fruit, date recognition, Gaussian mixture model, outlier detection.

\section{Introduction}

Date is a delicious fruit with a sweet flavor [1], which grows in many countries over the world. The main producers of dates are Middle East and South Africa countries [2]. Dates are consumed at their different maturity stages and in different manners. They are consumed in both fresh and dry forms [3], as such or together with other diets. In the processed form, dates are served as paste, jams, pickles, syrup and jellies [1] and [4-6].

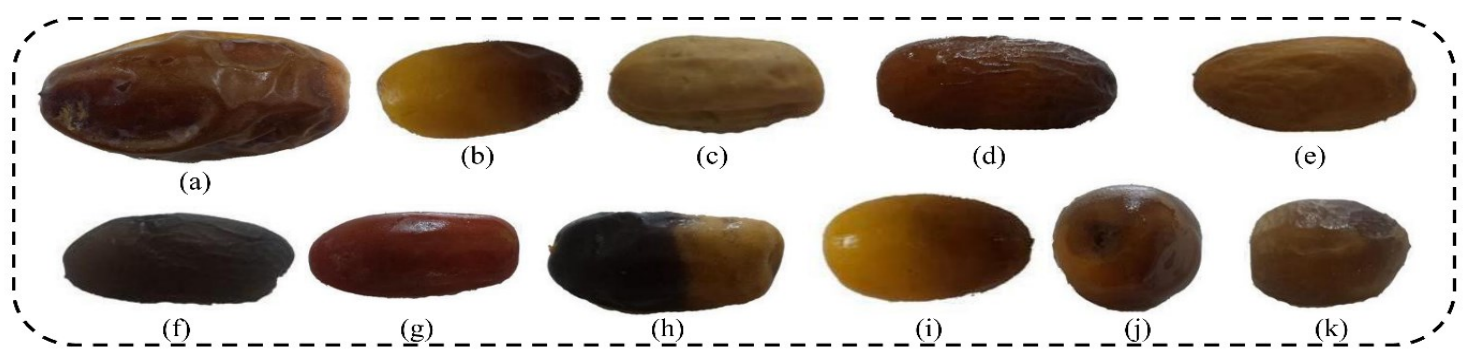

Fig. 1: Typical samples from the 11 varieties (a) Ajina, (b) Bayd hmam (c) Degla bayda (d) Bouaarous (e) Dfar lgat (f) Dgoul (g) Hamraya (h) Tinisin (i) Litima (j) Tantbucht and (k) Tarmount.

Correspondence to:o_aiadi@hotmail.com

Recommended for acceptance by Prof. Frédéric Lerasle

https://doi.org/10.5565/rev/elcvia.1041

ELCVIA ISSN: 1577-5097

Published by Computer Vision Center / Universitat Autonoma de Barcelona, Barcelona, Spain 
Besides, date fruit provides a wide range of interesting healthy and economic benefits. As for the healthy benefits of this high-value fruit, dates are considered as high energy food due to the significant quantity of sugars they contain [7]. Moreover, they contain an abundant amount of vitamins, minerals and dietary fibers [8]. Furthermore, dates are used to prevent the human body from several diseases including fever, memory disturbances and stomach disorders as well as chronic diseases such as cancer and heart diseases [9]. Economically, date industry significantly contributes to push the economy of producer countries, as all the parts constituting the date palm, including leaves and trunks, can be exploited for lucrative purposes [4].

Numerous date cultivars are planted through the world, resulting in several date varieties. Indeed, such a diversity allows date palm to fight against several dangerous diseases [3]. Date varieties are different in terms of color, texture, shape, taste and nutritional value [1] (Fig. 1). Even the samples belonging to the same variety may also considerably vary in terms of maturity stage, hardness degree and shape (Fig. 2(a), Fig. 2(b) and Fig. 2(c)). Fig. 1 shows typical images for samples from different varieties. Though the great nutritional, healthy and economic value of dates, many date varieties are no longer available in markets. This is because the culture about date fruit, including the noun of varieties, becomes less popular and starts to practically disappear from the daily discussions of people. Therefore, there is an urgent need to preserve such a valuable cultural heritage for the coming generations. In fact, successfully recognizing date samples is of great interest. For instance, diabetic subjects and those suffering from obesity need to recognize the varieties which they can consume and which leave their health status maintained [10]. Hence, with the remarkable advances in the field of computer vision, developing an automatic system for date recognition is strongly needed. Nevertheless, it is, sometimes, a very complicated task for a human to distinguish between some varieties, as they strongly resemble each other (Fig. 3(a), Fig. 3(b) and Fig. 3(c)). Computationally performing such a task is thus very challenging.

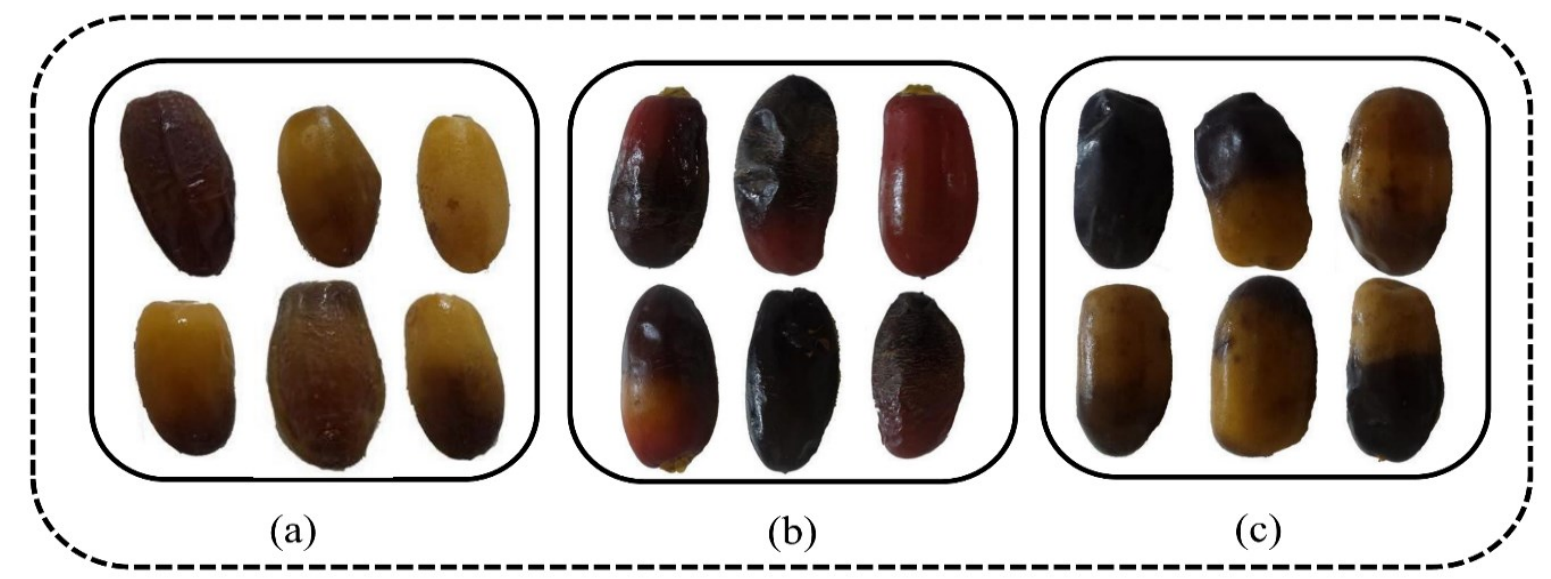

Fig. 2: Typical varieties with a large intra-variation. Samples within those varieties are different in terms of maturity stages, hardness degree and shape. (a) Bayd hmam (b) Hamraya and (c) Tinisin.

In the literature, much attention has been devoted to the task of visual object recognition in general [31], [32], [36], [37], [38], [39]. Certain methods have attempted to recognize different types of visual objects [31], [32], [33], while certain others have focused on recognizing a particular type of objects [34], [35], [36]. For instance, [40] proposed a probabilistic model, in which image features, dependencies between objects and outputs of local detectors, are incorporated to recognize different object categories. In [36], visual features are fused using a multiple kernel framework with SVM classifier in order to recognize the different flower categories. Fruits are particular type of objects, which have, in turn, attracted a considerable attention by researchers. For instance, In [41], artificial neural networks are used together with principal component to detect the defected surfaces on apple fruit. In [42], color and texture features were used to classify citrus peel diseases. In [43], physical features, including size and color, have been measured to classify peach fruit into three quality classes. 
State of the art studies concerned with date recognition can broadly be categorized into two categories. The first category includes studies that aim to classify date samples within a particular variety into distinct grades [11-14]. The second category includes methods that attempt to recognize for a given sample the variety it belongs to [15-17]. On one hand, date grading may be performed on the basis of defect percentage [13] and [18], quality [11] and [19] or maturity stage [20] and [12]. For instance, a method that uses color and size features to detect the cracked parts of dates is proposed in [13]. In [21], Back Propagation Neural Networks (BPNN) is used to classify date samples into three classes depending on the percentage of cracks within samples surface. The method in [22] separates acceptable from rejected samples using RGB-based color histogram features. A color mapping system was proposed by [18], rather than machine learning techniques, to evaluate date maturity and detect defects.

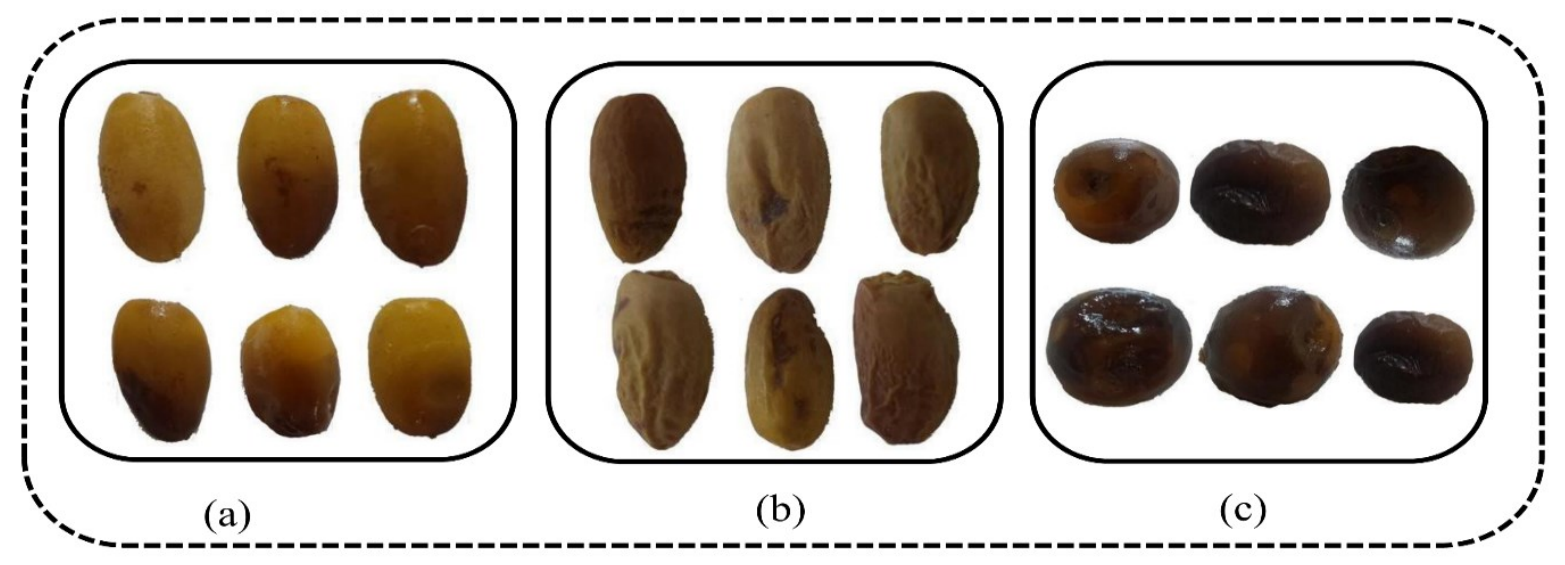

Fig. 3: Typical varieties with a small inter-variation, we can notice the high visual resemblance (a) Three samples from Bayd hmam and three others from Litima, (b) Three samples from Dfar lgat and three others from Degla bayda and (c) Three samples from Tarmount and three others from Tantbucht.

In some other studies, date samples have been graded according to their quality. Date quality can be measured according to several criterions including hardness, moisture and sugar content. For instance, dates were classified into dry, medium and moist on the basis of their moisture content [19]. Linear discriminate analysis (LDA) and stepwise discriminate analysis (SDA) were used to grade dates into hard, semi-hard and soft [11]. In [23], date samples were graded, based on hardness degree, into six grades using three classifiers, namely k-nearest neighbors (KNN), support vector machines (SVM) and LDA.

Other studies have focused on date grading based on the maturity stage. For instance, an HSVbased method was developed by [12] to grade date samples depending on their maturity stage. Similarly, in [20], a machine vision-based system was developed to grade Berhee variety into three maturity stages.

On the other hand, studies concerned with the recognition of different date varieties are very scarce. The method of [15] utilized texture features namely, Weber Law Descriptors (WLD) and Local Binary Patterns (LBP) to recognize four date varieties. In [24], physical measurements including moisture content and water activity were taken from each date sample. These measurements were then incorporated with color features and fed to a neural network classifier in order to recognize date samples from seven varieties. Using such measurements is, in fact, impractical and makes very difficult to automate the method. In [16] color, shape and texture descriptors were extracted so as to recognize seven date varieties. As classifiers, KNN, LDA and Artificial Neural Network (ANN) were employed, while the ANN has outperformed the others. Nevertheless, [16] reported that the ANN has 
taken a great deal of time before yielding the optimal network. Such an expensive cost is amongst the main barriers to meet the requirements of an automatic recognition system. In [17], Five varieties were recognized using probabilistic neural networks (PNN) fed by color properties.

It is worth noting that in the previous studies many fundamental issues are either not appropriately handled or even totally ignored, these issues are:

1- Recognizing only fully mature dates, however, date samples could be in different maturity stages when it being recognized. In fact, difference in maturity stage implies that samples belonging to the same variety are significantly distinct in terms of color, which makes the process of recognition more complicated.

2- Some of those studies used methods which are computationally expensive, which does not meet the requirements of a real-time recognition system.

3- In some of those studies, some physical measurements from dates (e.g., moisture content) are needed to perform the recognition task. Such a method can hardly be automated.

4- To the best of our knowledge, none of the existing methods take into account the existence of outlier samples. An outlier sample can be either a deformed one (e.g., because of the transportation conditions) or much grown one compared to its corresponding ones or a sample with visual characteristics that deviate from those of the related samples.

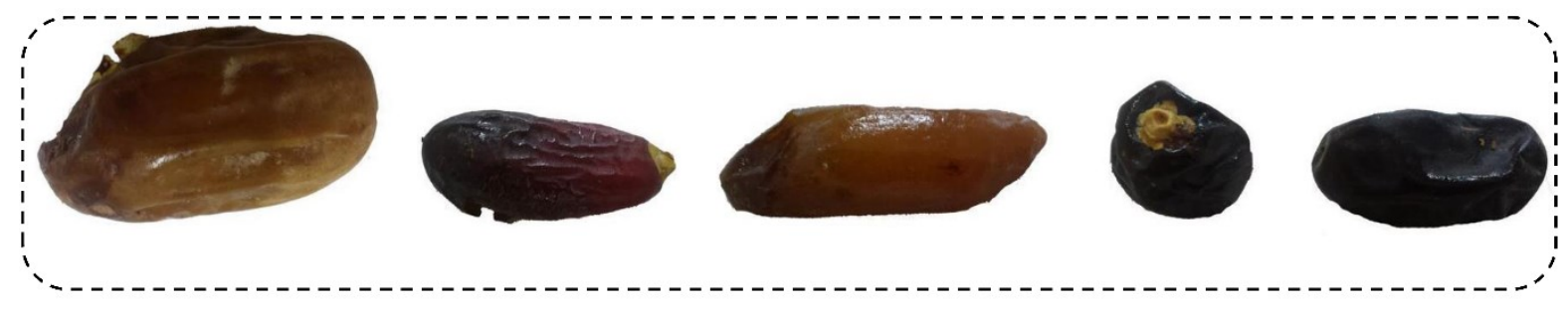

Fig. 4: Some outlier samples from different varieties.

In our work, however, we solve these issues in order to improve the task of date fruit recognition. At first, we collect date samples from 11 varieties, and then we take images for them. We divide those images into two sets: training and testing. Image features describing the different aspects of images were then extracted from all the images. Because the training set may contain some outlier samples which could degrade the quality of recognition outcomes, we prune this set from those outliers. To do so, we use the Pruning Local Distance-based Outlier Factor (PLDOF) method proposed in [25], where training images, related to each variety, are pruned separately. In the PLDOF, samples are firstly clustered and then outliers are removed from each cluster, where the number of clusters is manually tuned. Thus, in order to make our method fully automatic, we use Davies-Bouldin index to automatically and accurately determine the optimal number of clusters. Each of these clusters is assumed to be normally distributed, where this assumption is checked using Mardia's multivariate skewness and kurtosis tests [26]. Afterwards, we combine the probability density functions that correspond to each cluster in a Gaussian Mixture Model (GMM) representing the visual model of the date variety. It is worth noting that we use the Expectation-Maximization (EM) algorithm for parameters estimation. Then, to recognize a new sample, we calculate the probability that the sample belongs to each variety. This probability is equal to the sum of likelihoods that the sample belongs to each component, within the variety mixture, multiplied by the component weight. The sample is assigned to the variety having obtained the highest score.

The proposed method makes several noteworthy contributions to the literature. First, it is fully automatic, as neither physical measurements nor any kind of human assistance are needed. Second, it reaches a high recognition rate in spite of the existence of the outlier samples. Third, despite the noticeable difference, in terms of maturity stage, hardness degree and shape, between samples 
appertaining to the same variety, our method successfully recognize them. Moreover, it is capable to distinguish between the strongly confusing varieties. Fourth, it is computationally fast, as we will show in the experiments section. Experimental results, given at the end of this paper, show that our method outperforms the previous methods in terms of recognition accuracy.

The remainder of this paper is organized as follows: In Section 2, we give details about our benchmark. Section 3 describes the image acquisition system that we have used. Section 4 describes the proposed method. The experimental results are presented in Section 5. Finally, we draw some conclusions in Section 6.

\section{Samples Collection}

In order to investigate the performance of the proposed method and since no date benchmark is publicly available, we introduce a new benchmark that includes 11 varieties. These varieties are Ajina, Bayd hmam, Bouaarous, Degla bayda, Dfar lgat, Dgoul, Litima, Hamraya, Tarmount, Tantbucht and Tinisin, respectively. These varieties are collected from the local markets of Touggourt region (south Algeria), in which some experienced farmers sell different date cultivars during the months of September and October every year. The total number of samples collected was 660 (i.e., 60 samples for each variety). Fig. 1 shows typical samples from each variety.

Three main properties make our benchmark different than the ones used in the previous studies, which are:

1- It includes the highest number of varieties (11) compared to the related studies. Typical samples from the 11 varieties are shown in Fig. 1.

2- It includes some varieties with a large intra-variation, as they comprise samples in the different maturity stages (i.e., immature, semi-mature, fully mature), hardness degree and shape (Fig. 2(a), Fig. 2(b) and Fig. 2(c)). Besides, it contains some varieties with a small intervariation because they highly resemble each other, as shown in Fig. 3(a), Fig. 3(b) and Fig. 3(c).

3- It contains outlier samples such as those deformed because of the transportation conditions and those which grew much more compared to their corresponding ones (Fig. 4).

\section{Image Acquisition system}

In this work, we have opted for the following image acquisition system

- A white background.

- A Samsung camera at a resolution of 4128x3096 pixels.

- The camera has been fixed at a distance of $30 \mathrm{~cm}$ from the white background.

- Regarding lighting, we have used two standard fluorescent lights.

- Images were taken in a dark chamber, where the sample being imaged is illuminated with the two fluorescent lights.

- The process of the acquisition consists in manually placing a sample on the white background, shooting it, then replacing it with the next one.

- The obtained images have been represented and saved using RGB color space.

- Images were then manually refined to remove some noise and bad-illuminations. 


\section{Proposed Method}

The main concern of this study is to automatically recognize different date varieties using machine learning techniques. In this section, we provide details about the proposed method. Fig. 5 depicts the steps of the proposed method.

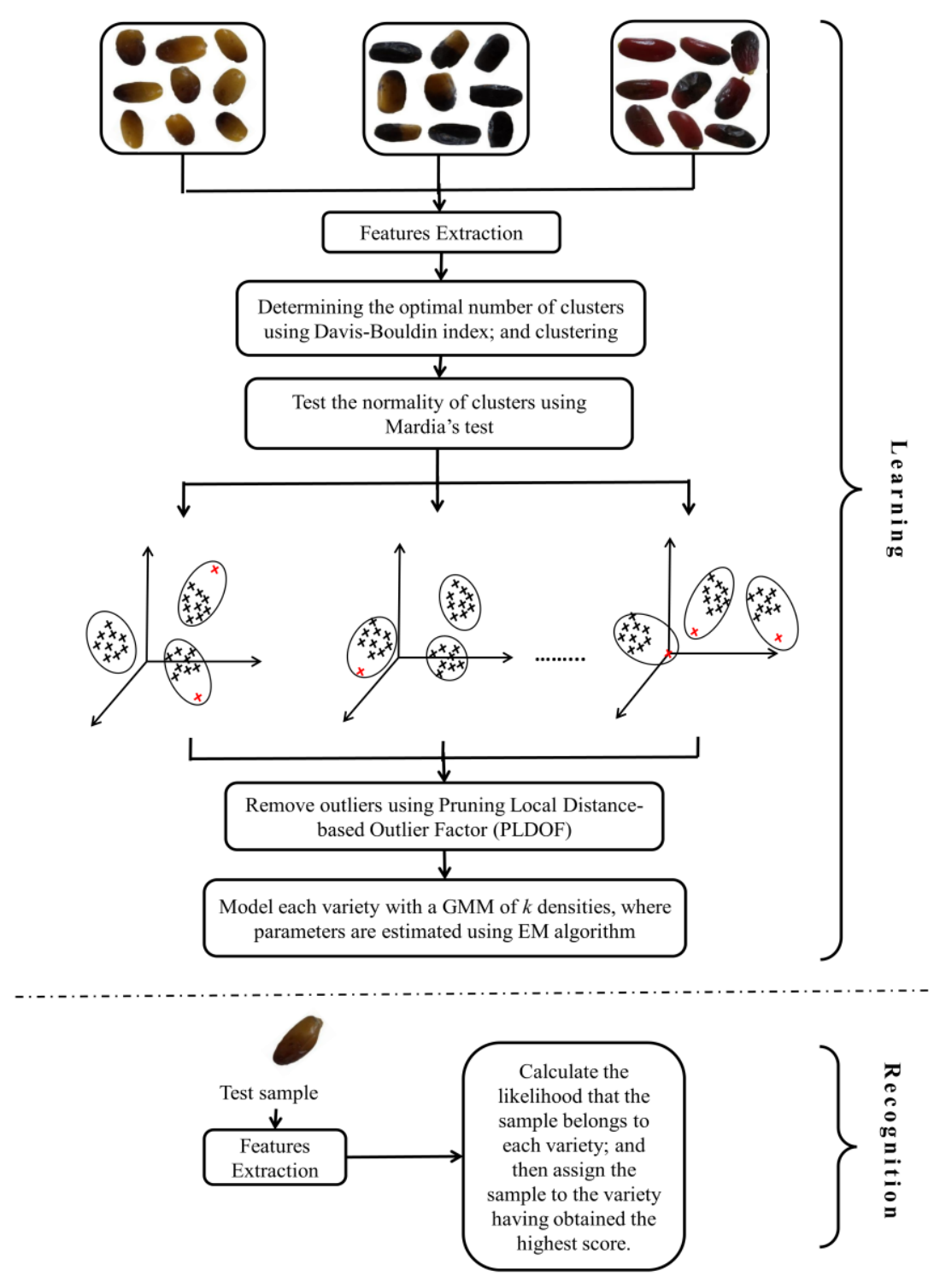

Fig.5: The steps of the proposed method for automatic date fruit recognition.

\subsection{Features Extraction}

Feature selection is amongst the most challenging tasks in pattern recognition problems [27]. This is because any mis-selection could potentially lead to negatively affect the recognition outcomes. Hereafter, we give an overview on the used features, including color, shape and texture features.

\subsubsection{Color features}

Color is one of the most important features used to describe image content [28]. From Fig. 1, we can see that there is a difference in color between date samples from the different varieties. For this 
reason, color would be very useful in distinguishing two distinct samples. Color histogram describes the percentage of colors within an image, where each bin in the histogram represents the appearance frequency of the corresponding color. We have noticed that date fruits have a very limited number of color variations. Thus and instead of using a universal color quantization, we have opted for a more reliable mapping scheme. Firstly, each color channel from the RGB space is quantized into 12 bins. Then, all images of the dataset are mapped into a new sub-space that consists in $144 * \mathrm{QR}+12 * \mathrm{QG}+\mathrm{QB}=1727$ bin, where $\mathrm{QR}, \mathrm{QG}$ and $\mathrm{QB}$ results from quantizing $\mathrm{R}, \mathrm{G}$ and $\mathrm{B}$, respectively. After having all images mapped to this new space, a histogram $H$ of 12727 length is calculated where $H(i)$ represents the number of pixels, from all the dataset, that falls in bin $i$. Fig. 6 illustrates the obtained histogram $H$.

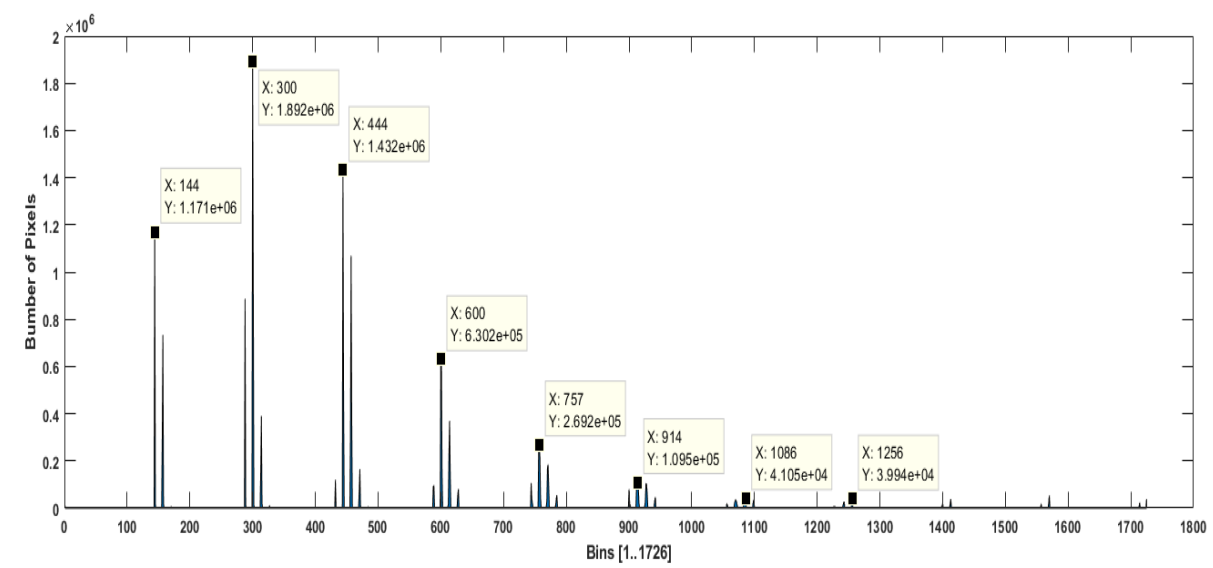

Fig. 6: Color histogram of images dataset.

From the Fig. 6, we can see that the histogram $H$ has a set of leaps. These leaps represents where the majority of pixels are mapped. For example, we can see that the majority of pixels falls in the bin 300 which corresponds to $\mathrm{QR}=11, \mathrm{QG}=2$ and $\mathrm{QB}=0$ (i.e, $11 * 144+12 * 2+0=300$ ). Based on this result, we have found that a compact and more representative histogram $H^{\prime}$ of length 29 , that represents the dense bins, can be drawn from the original 1727 histogram $H$.

Color histogram presents diverse pros: it can easily be implemented; it is straightforward and efficient; it is invariant to translation and rotation changes [28]. Nevertheless, color histogram lacks the ability of describing color spatial distribution (i.e., how colors are arranged in the image). Color distribution within the sample surface is of great importance and could provide an indication about the variety to which the sample belongs. Hence, for each image, we extract color standard deviation from each of the three RGB channels, as in Equation 1.

$$
\text { Standard deviation }=\sqrt{\frac{1}{N-1} \sum_{i=1}^{N}\left(V_{i}-\mu\right)^{2}}
$$

where $N$ is the number of pixels in the image and $V_{i}$ is the pixel value in one of the three RGB channels. $\mu$ is the mean of values in one channel.

\subsubsection{Texture features}

Two samples that belong to two different varieties may noticeably resemble each other in terms of color. Hence, color should be concatenated with other features in order to assure a high discrimination power. Texture of some samples is smooth, whereas this is not the case with some others. Thus, taking into consideration such an interesting aspect is quite important and could help us in accurately recognizing date samples. We use the Gray-Level Co-occurrence Matrix (GLCM) [29], which 
represents the appearance frequency of gray-level pairs within an image, as a texture descriptor. More formally, suppose we are given a GLCM, referred to as $M$, then, each entry $M(i, j)$ represents the occurrence frequency of a gray-level $j$ followed by a gray-level $i$ according to a spatial relationship, denoted as $S(\Delta x, \Delta y)$.

Given an image $I$ and a spatial relationship $S(\Delta x, \Delta y), N$ is calculated according to Equation 2 .

$$
M(i, j)=\sum_{p=1}^{H} \sum_{q=1}^{W} \begin{cases}1, & \text { if } I(p, q)=i \text { and } I(p+\Delta x, q+\Delta y)=j \\ 0, & \text { otherwise }\end{cases}
$$

where $H$ and $W$ are respectively the height and the width of $I$.

In order to reduce the matrix dimensions, the gray-level channel is divided into 8 equal ranges. After having investigated several spatial relationships, we consider four relationships: $S(0,1)$, $S(-1,1), S(1,0)$ and $S(1,1)$. The final matrix $M$ is the sum of the four matrices extracted according to those spatial relationships.

After having calculated $M$ and normalize it (such that sum of the elements equal to 1), four features are extracted from this matrix. These features are given by Equation 3-6.

$$
\begin{aligned}
& \text { Contrast }=\sum_{i} \sum_{j}(i-j)^{2} M(i, j) \\
& \text { Correlation }=\frac{\sum_{i} \Sigma_{j}\left(i-\mu_{x}\right)\left(i-\mu_{y}\right) M(i, j)}{\sigma_{x} \sigma_{y}} \\
& \text { Energy }=\sum_{i} \sum_{j} M(i, j)^{2} \\
& \text { Homogenteiy }=\sum_{i} \sum_{j} \frac{1}{1+(i-j)^{2}} M(i, j)
\end{aligned}
$$

Where $\mu_{x}, \sigma_{x}$ respectively are the mean and standard deviation of the marginal probability matrix $M_{x}(i)=\sum_{j} M(i, j) \cdot \mu_{y}, \sigma_{y}$ respectively are the mean and standard deviation of the marginal probability matrix $M_{y}(j)=\sum_{i} M(i, j)$.

\subsubsection{Shape features}

Shape is a quite important feature because there are some varieties which can easily be distinguished from their shapes. For instance, Tantbucht has roughly a spherical form, whereas, Dgoul has a cylindrical form. Meanwhile, it should be mentioned that using only shape features could probably lead to confuse some varieties with others. In this work, the shape features we used are the following:

1. Minor axis length: it is the shortest diameter within a sample surface.

2. Major axis length: it is the longest diameter within a sample surface; it is perpendicular to the major axis.

3. Eccentricity: it is the ratio of the major axis to the minor axis. It is used to measure the slimness of the sample.

4. Area: it is the number of pixels constituting the date sample surface.

5. Perimeter: it is the number of pixels at the margin of the date sample.

In order to calculate the minor, major axis length and eccentricity, we use the principal axes method. First, canny edge detector is applied to each image from the dataset where images are converted to gray level before feeding them to the detector. After that, the covariance matrix describing the spread of edge points is calculated (for each image separately), where the resulting matrix is of $2 \times 2$ dimensions. 
Let $C$ be a covariance matrix of the form

$$
C=\left(\begin{array}{l}
C_{11} C_{12} \\
C_{21} C_{22}
\end{array}\right)
$$

The eigenvalues of $C$, denoted as $\lambda$, can be calculated as in Equation 8 .

$$
(C-\lambda \cdot I) \cdot v=0
$$

such that $v$ is non-zero vector and $I$ is the identity matrix.

Taking into consideration that $v$ is non-zero vector, Equation 8 is satisfied if $|C-\lambda . I|=0$,

$$
|C-\lambda . I|=\left|\begin{array}{cc}
C_{11}-\lambda & C_{12} \\
C_{21} & C_{22}-\lambda
\end{array}\right|=\left(C_{11}-\lambda\right)\left(C_{22}-\lambda\right)-C_{12} C_{21}=0
$$

Thus

$$
\left\{\begin{array}{l}
\lambda 1=\frac{1}{2}\left(C_{11}+C_{22}+\sqrt{\left(C_{11}+C_{22}\right)^{2}-4\left(C_{11} C_{22}-C_{12} C_{21}\right)}\right) \\
\lambda 2=\frac{1}{2}\left(C_{11}+C_{22}-\sqrt{\left(C_{11}+C_{22}\right)^{2}-4\left(C_{11} C_{22}-C_{12} C_{21}\right)}\right.
\end{array}\right.
$$

The highest value from $\lambda_{1}$ and $\lambda_{2}$ corresponds to the major axis length, while the lowest one corresponds to the minor axis length. Let $\lambda_{1}$ be the major axis and $\lambda_{2}$ be the minor axis length, the eccentricity is given by Equation 11 .

$$
E=\frac{\frac{\lambda_{1}}{\lambda_{2}}-E_{\min }}{E_{\max }-E_{\min }}
$$

Where $E_{\min }$ is the minimum eccentricity for all the samples and $E_{\max }$ the minimum eccentricity for all the samples.

After the features having extracted, features dimensions were reduced to 10 using Principal Component Analysis (PCA).

\subsection{Pruning samples from outliers}

As reported above, training samples could include some outliers which could negatively affect the recognition outcomes. Hence, in order to avoid falling in such a problem, we prune training samples from the outlier ones. For this end, we use the Pruning Local Distance-based Outlier Factor (PLDOF) method proposed in [25]. The core idea of the PDLOF is the calculation of a measure, which is called Local Distance-based Outlier Factor (LDOF), for some specific samples. A high LDOF value for a sample indicates that it deviates from its neighbors and it is most likely to be an outlier.

Formally, let $X=\left\{x_{i}, i=1, \ldots, T\right\}$ be a set of data points in the M-dimensional space. Let $N_{x_{i}}$ be the set of K-nearest neighbors of $x_{i}$, LDOF is defined as $\operatorname{LDOF}\left(x_{i}\right)=\overline{d_{p}} / \overline{D_{p}}$, where $\overline{d_{p}}$ represents the average distance from $x_{i}$ to the data points in $N_{x_{i}}$, it is given by Equation 12 . 


$$
\overline{d_{p}}=\frac{1}{\left|N_{x_{i}}\right|} \sum_{q \in N_{x_{i}}} d\left(x_{i}, q\right)
$$

where $\left|N_{x_{i}}\right|$ is the magnitude of $N_{x_{i}}$ and $d\left(x_{i}, q\right)$ is the distance between $x_{i}$ and the data point $q \epsilon N_{x_{i}}$. $\overline{D_{p}}$ represents the average distance among data points in $N_{x_{i}}$, it is defined by Equation 13.

$$
\overline{D_{p}}=\frac{1}{\left.\left|N_{x_{i}}\right|\left|N_{x_{i}}\right|-1\right)} \sum_{q, q^{\prime} \in N_{x_{i}}, q \neq q^{\prime}} d\left(q, q^{\prime}\right)
$$

The remaining steps of the algorithm are summarized in the Algorithm1.

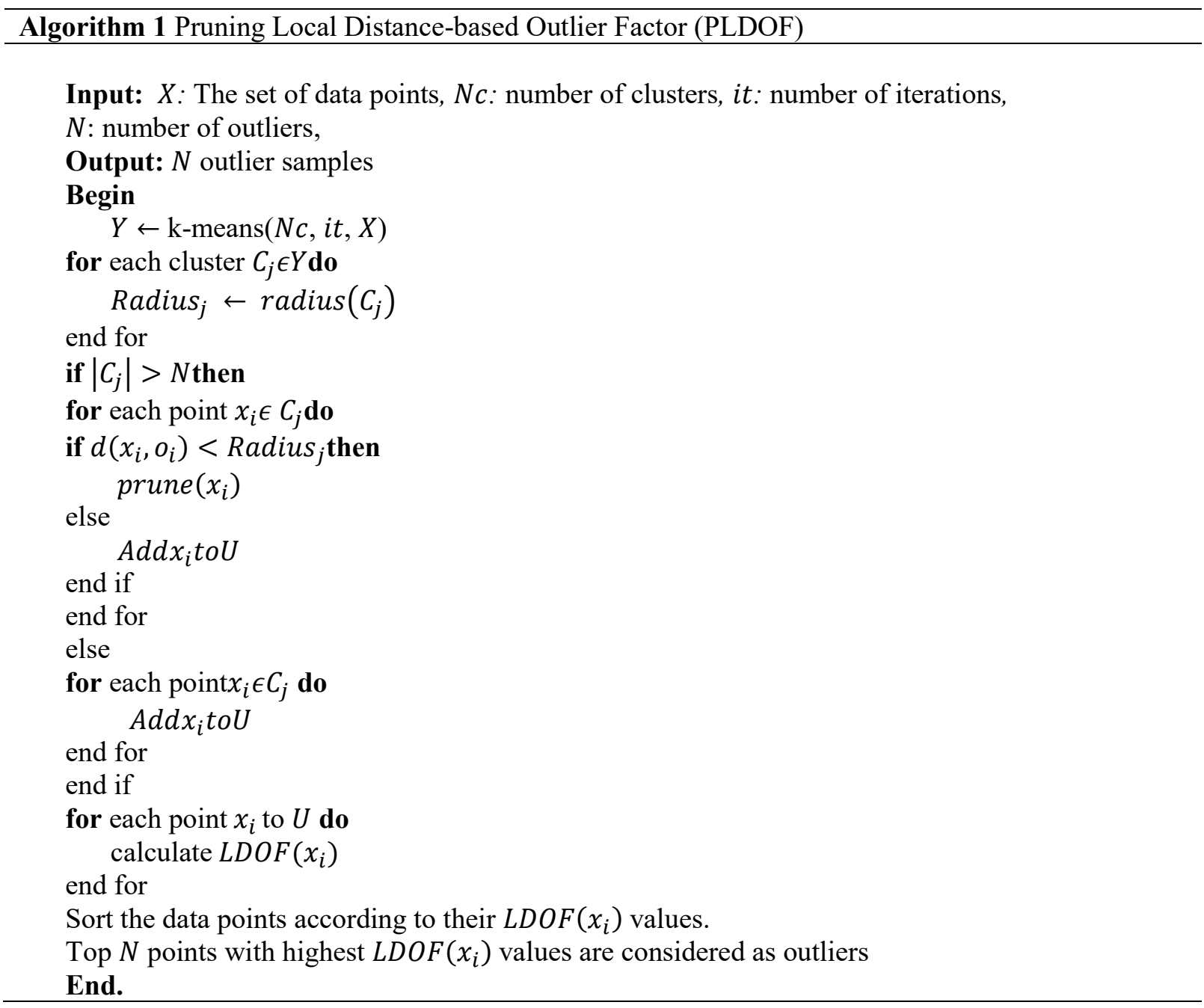

where $o_{i}$ and Radius $_{j}$ are the centroid of the cluster $C_{j}$ and its radius, respectively.

The PDLOF algorithm is applied to each element in the set $X^{\prime}=\left\{X_{i}, i=1, \ldots, 11\right\}$, where $X_{i}$ is the set of training samples of the $i^{t h}$ variety. The number of outliers removed from each variety is 5 , where the number of neighbors considered in calculating the LDOF values is empirically set to 9.

\subsection{Determining the optimal number of clusters using Davies-Bouldin index}

In the PDLOF method, the number of clusters is required to be supplied as an input parameter. In fact, the number of clusters may significantly vary from a variety to another because there is a 
prominent difference between the visual characteristics of samples affiliated to distinct varieties. Thus, abusively fixing a unique value for all the varieties is not suitable for our case. In this work, therefore, we propose to automatically determine the optimal number of clusters for each variety using DaviesBouldin index [30]. The idea is to evaluate the index for different values of the number of clusters, with the aim of comparing clustering solutions. The minimum value of the index indicates the preferred solution.

Suppose we are given a set $X=\left\{x_{i}, i=1, \ldots, T\right\}$, once $X$ is clustered into $K$ clusters we get the set $C=\left\{c_{i}, i=1, \ldots, K\right\}$. Let us denote also by Equation 14 .

$$
\bar{c}_{i}=\frac{\sum_{j=1, x_{j} \in c_{i}}^{T} x_{j}}{\left|c_{i}\right|}
$$

the centroid of the cluster $c_{i}$.

Davies-Bouldin index is defined as Equation 15.

$$
D B(K)=\frac{1}{K} \sum_{i=1}^{K} V_{i}
$$

where

$$
V_{i}=\max _{j=1, \ldots, K, j \neq i}\left(\frac{\delta_{i}+\delta_{j}}{d\left(\bar{c}_{i}, \bar{c}_{j}\right)}\right)
$$

where $\delta_{i}$ and $\delta_{j}$ are the average distance of all data points in the clusters $c_{i}$ and $c_{j}$ to their cluster centroids $\bar{c}_{i}$ and $\bar{c}_{j}$, respectively, and $d\left(\bar{c}_{i}, \bar{c}_{j}\right)$ is the distance between these two latter centroids. $\delta_{i}$ (respectively $\delta_{j}$ ) is given by

$$
\delta_{i}=\frac{\Sigma_{t=1}^{\left|c_{i}\right|}\left\|x_{t}-\bar{c}_{i}\right\|}{\left|c_{i}\right|}
$$

and $d\left(\bar{c}_{i}, \bar{c}_{j}\right)$ is given by

$$
d\left(\bar{c}_{i}, \bar{c}_{j}\right)=\left\|\bar{c}_{i}-\bar{c}_{j}\right\|
$$

In order to detect the optimal number of clusters for each variety $V$, we, separately, applied Davies-Bouldin index to the different training sets related to those varieties, where $K=\{3, \ldots, 9\}$. At the end of this step, for each variety $V$, we got the set of clusters $C_{V}^{\prime}=\left\{c^{\prime}{ }_{V_{k}}, k=1, \ldots, k_{V}^{\prime}\right\}$ where each cluster includes visually similar samples. Table 1 shows the optimal number of varieties $k_{V}^{\prime}$ detected for each variety $V$.

Table 1: The optimal number of clusters detected for each variety.

\begin{tabular}{|l|l|}
\hline Variety & $\begin{array}{l}\text { Optimal number } \\
\text { of clusters }\end{array}$ \\
\hline Ajina & 9 \\
\hline Bayd hmam & 6 \\
\hline
\end{tabular}




\begin{tabular}{|l|l|}
\hline Bouaarous & 9 \\
\hline Degla bayda & 5 \\
\hline Dfar lgat & 9 \\
\hline Dgoul & 9 \\
\hline Hamraya & 9 \\
\hline Litima & 4 \\
\hline Tantbucht & 7 \\
\hline Tarmount & 6 \\
\hline Tinisin & 9 \\
\hline
\end{tabular}

In order to detect the optimal number of clusters, clustering has been conducted based on the features we have extracted namely color, shape and texture. By seeing the yielded values for the different varieties, we can make some observations. For instance, for Tinisin, the optimal number is 9 , this is could be attributed to the fact that samples belonging to this variety are in different maturity level (i.e., different in terms of color). One reason behind the difference in maturity level could be the age of prune. Samples in the beginning of the harvesting season are immature and fully mature at the end. We can note also that the optimal number for Ajina is 9, despite that samples of this variety are almost homogeneous in terms of color. This may be explained by the fact that Ajina samples are different in terms of shape and texture as well. Certain samples have a smooth texture, whereas certain others have a random texture.

\subsection{Testing the normality of clusters}

Since visually similar images are grouped in the same cluster, we assume that samples within each cluster are normally distributed. We check this assumption using Mardia's multivariate skewness and kurtosis tests [26]. Formally, suppose we are given a cluster $c^{\prime}{ }_{V_{k}}=\left\{x_{i}^{\prime}, i=1, \ldots, P\right\}$, where $x_{i}^{\prime}$ is a Mdimensional sample data point that belong to ${c^{\prime}}_{V_{k}}$. Let $\mu$ and $\sum$ denote the mean of samples belonging to $c^{\prime}{ }_{V_{k}}$ and the samples covariance matrix, respectively. $\mu$ and $\sum$ are defined as

$$
\begin{gathered}
\mu=\frac{1}{P} \sum_{i=1}^{P} x^{\prime}{ }_{i} \\
\sum=\frac{1}{P} \sum_{i=1}^{P}\left(x^{\prime}{ }_{i}-\mu\right)\left(x^{\prime}{ }_{i}-\mu\right)^{\prime}
\end{gathered}
$$

Mardia multivariate skewness and kurtosis are defined as follows:

$$
\begin{gathered}
\beta_{2, M}=\frac{1}{P} \sum_{i=1}^{P}\left(x_{j}^{\prime}-\mu\right)^{\prime} \Sigma^{-1}\left(x_{j}^{\prime}-\mu\right)^{2} \\
\beta_{1, M}=\frac{1}{P^{2}} \sum_{i=1}^{P} \sum_{j=1}^{P}\left(x^{\prime}{ }_{j}-\mu\right)^{\prime} \Sigma^{-1}\left(x^{\prime}{ }_{j}-\mu\right)^{3}
\end{gathered}
$$

For a multivariate normal distribution:

1- The test statistic:

$$
Z_{1, M}=\frac{P}{6} \beta_{1, M}
$$


follows a chi-square distribution with $f=\frac{1}{6} M(M+1)(M+2)$ degrees of freedom.

2- The test statistic:

$$
Z_{1, M}=\frac{\beta_{1, M}-M(M+2)}{\sqrt{8 M(M+2) / P}}
$$

follows a standard normal distribution.

According to Mardia's test, clusters within the set $C_{V}^{\prime}=\left\{c^{\prime}{ }_{k^{\prime}}, k=1, \ldots, k_{V}^{\prime}\right\}$ where $V=1, \ldots, 11$ are found to be normally distributed.

\subsection{Date variety modeling using Gaussian mixture model (GMM)}

Each cluster within $C_{V}^{\prime}$ is normally distributed. Therefore, it is described by the probability density of an M-dimensional normal function (pdf) of the form

$$
G\left(x \mid \theta_{k}\right)=\frac{1}{\sqrt{\left|\sum_{k}\right|(2 \pi)^{M}}} e^{-\frac{1}{2}\left(x-\mu_{k}\right)^{T} \sum^{-1}\left(x-\mu_{k}\right)}
$$

where $x$ is M-dimensional data vector that belongs to the set $X$, and $\theta_{k}$ denotes the parameters of the Gaussian distribution that corresponds to the $k^{\text {th }}$ cluster, these parameters are

$\mu_{k}$ : The mean vector of the points belonging to the cluster.

$\sum_{k}$ : The covariance matrix.

Probability density functions (pdf) $G_{V}=\left\{G_{V_{k}}, k=1, \ldots, k_{V}^{\prime}\right\}$, which correspond to the set of clusters $C_{V}^{\prime}=\left\{c^{\prime}{ }_{V_{k}}, k=1, \ldots, k_{V}^{\prime}\right\}$ of a date variety $V$, were combined in a Gaussian Mixture Model (GMM) representing the visual model of this variety. Thus, our model is a GMM, which is a weighted sum of $k_{V}^{\prime}$ component Gaussian densities, it is given by

$$
P_{V}\left(x \mid \theta_{V}\right)=\sum_{k=1}^{k_{V}^{\prime}} w_{k} G\left(x / \theta_{V_{k}}\right), \theta_{V}=\left\{\theta_{V_{\mathbf{k}}}, k=1, \ldots, k_{V}^{\prime}\right\}
$$

where $w_{k}$ denotes the weight of the $k^{\text {th }}$ distribution, and $\theta_{V}$ the parameters of Gaussian component densities of the mixture $P_{V}$. The likelihood of the data that belong to $C_{V}^{\prime}$ is given by

$$
L=\prod_{i=1}^{N_{V}} P_{V}\left(x \mid \theta_{V}\right)
$$

where $N_{V}$ denotes the number of data points that belong to $C_{V}^{\prime}$.

Expectation-Maximization (EM) algorithm is then used to maximize $L$ and estimate the parameters of $\theta_{V}$

1. Expectation step 


$$
y_{i j}=\frac{\frac{w_{j}}{\sqrt{|\Sigma|(2 \pi)^{M}}} e^{-\frac{1}{2}\left(x_{i}-\mu_{j}\right)^{T} \Sigma^{-1}\left(x_{i}-\mu_{j}\right)}}{\sum_{i=1}^{N_{V}} y_{i j}}
$$

2. Maximization step

$$
\begin{gathered}
w_{j}=\frac{\sum_{i=1}^{N_{V}} y_{i j}}{N_{V}} \\
\mu_{j}=\frac{\sum_{i=1}^{N_{V}} x_{i} y_{i j}}{\sum_{i=1}^{N_{V}} y_{i j}} \\
\sum_{j}=\frac{\sum_{i=1}^{N_{V}} y_{i j}\left(x_{i}-\mu_{j}\right)\left(x_{i}-\mu_{j}\right)^{T}}{\sum_{i=1}^{N_{V}} y_{i j}}
\end{gathered}
$$

These two steps are repeated for $i=1, \ldots, N_{V}$ and $j=1, \ldots, k_{V}^{\prime}$ until convergence.

\subsection{Recognition of new samples}

The steps we follow to recognize a test sample are summarized in Algorithm 2.

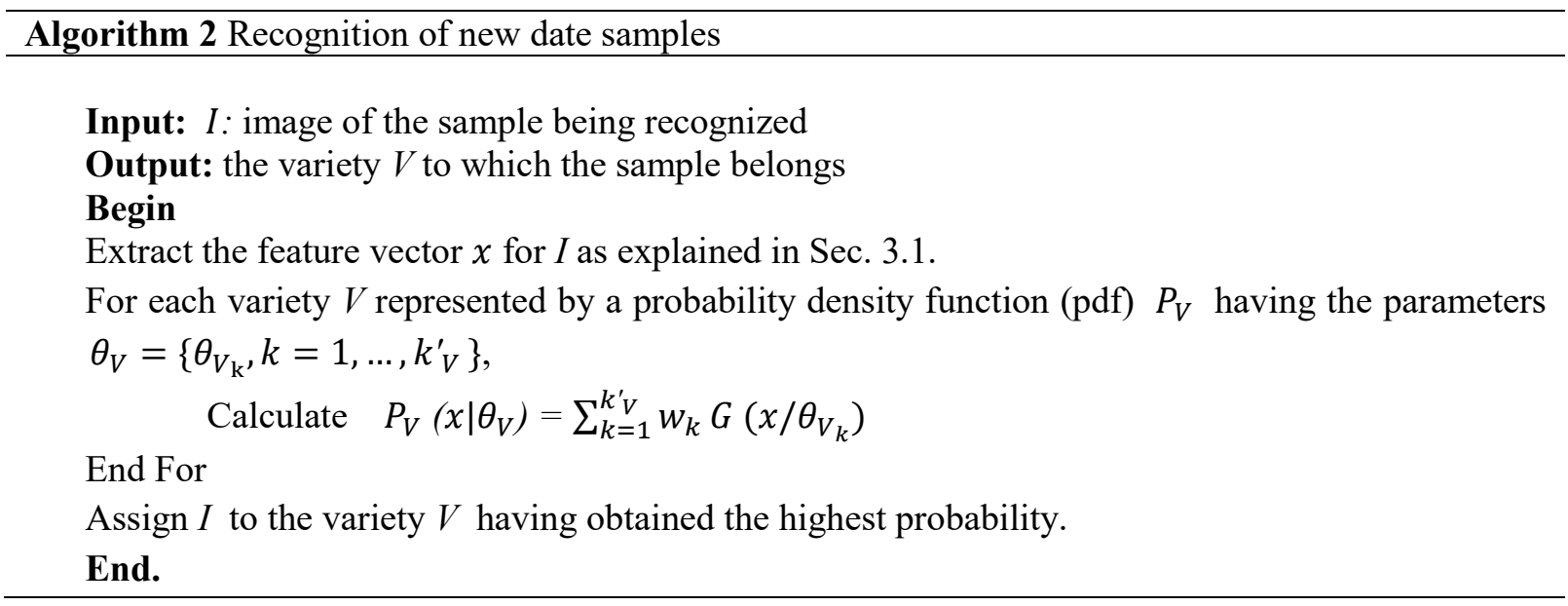

\section{Experiments}

To assess the performance of the proposed method, we carry out five experiments, each of which is concerned with a particular aspect. In the first experiment, we evaluate the overall performance of our proposed method. The aim of the second experiment is to measure the processing time required for recognition. The third experiment is devoted to compare our method with three other methods from the state of the art. A fourth experiment is dedicated to test the influence of each of the feature combinations on the recognition outcomes. Finally, a last experiment was conducted in order to compare the performance of the proposed method against the human performance. Before going ahead in reporting our findings, let us first review the experimental setup for evaluating the present method. 


\subsection{Experimental setup}

\subsubsection{Dataset}

All the experiments were conducted on the collected dataset described in Section 2.

\subsubsection{Performance measures}

To make a fair comparison with the state of the art methods, we adopt the recognition accuracy as a performance measure. The recognition accuracy for a single variety is defined as:

$$
\text { Accuracy }_{v}=\frac{\text { Number of correctly recognized samples }}{\text { Number of samples assigned to the variety }} \times 100
$$

The average recognition accuracy over all the varieties is given by

$$
\text { Accuracy }_{o}=\frac{\sum_{1}^{N} \text { Accuracy }_{v}}{N},
$$

where $N$ is the number of varieties.

\subsubsection{Experimental conditions}

As for the hardware used to perform the experiments, an Intel Core i5-2410 processor with a speed of $2.30 \mathrm{GHz}$ is used along with a memory (RAM) of $4 \mathrm{~GB}$. The experiments were carried out under a Windows 7 Professional 32 bits system.

\subsection{Experimental results}

\subsubsection{First experiment}

By referring to Fig. 5, we can see that the proposed method includes two main stages, namely learning and testing. The learning stage, in turn, includes three stages which are features extraction, detecting the optimal number of clusters, eliminating outliers and modeling using the GMM. After the learning process having finished, the testing process is launched in order to assign each test sample to the suitable variety. The present experiment aims to determine the recognition accuracy that can be achieved by the proposed method. To reach such an aim, we follow the steps shown in Algorithm 2. In all the experiments, a 3-fold cross-validation technique is adopted. In one iteration, two folds are used for training, while the rest is devoted for testing. Thus, after 3 iterations, all the samples are tested. For the sake of clarity, we summarize our findings in the confusion matrix shown in Table 2. 
Table 2: The confusion matrix.

\begin{tabular}{|c|c|c|c|c|c|c|c|c|c|c|c|c|}
\hline Output & : & 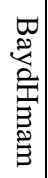 & 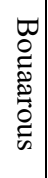 & 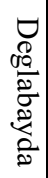 & 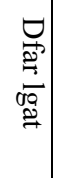 & 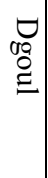 & & 冚 & 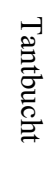 & 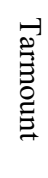 & : & \\
\hline Ajina & 20 & 0 & 0 & 0 & 0 & 0 & 0 & 0 & 0 & 0 & 0 & $100 \%$ \\
\hline Bayd hmam & 0 & 20 & 0 & 0 & 0 & 0 & 0 & 1 & 0 & 0 & 0 & $95.2 \%$ \\
\hline Bouaarous & 0 & 0 & 19 & 0 & 0 & 0 & 1 & 0 & 0 & 0 & 0 & $95 \%$ \\
\hline Degla Bayda & 0 & 0 & 0 & 20 & 0 & 0 & 0 & 0 & 0 & 0 & 0 & $100 \%$ \\
\hline Dfar lgat & 0 & 0 & 0 & 0 & 20 & 0 & 0 & 0 & 0 & 0 & 0 & $100 \%$ \\
\hline Dgoul & 0 & 0 & 0 & 0 & 0 & 20 & 0 & 0 & 0 & 0 & 0 & $100 \%$ \\
\hline Hamraya & 0 & 0 & 1 & 0 & 0 & 0 & 19 & 0 & 0 & 0 & 0 & $95 \%$ \\
\hline Litima & 0 & 0 & 0 & 0 & 0 & 0 & 0 & 19 & 0 & 0 & 0 & $100 \%$ \\
\hline Tantbucht & 0 & 0 & 0 & 0 & 0 & 0 & 0 & 0 & 20 & 0 & 0 & $100 \%$ \\
\hline Tarmount & 0 & 0 & 0 & 0 & 0 & 0 & 0 & 0 & 0 & 20 & 0 & $100 \%$ \\
\hline Tinisin & 0 & 0 & 0 & 0 & 0 & 0 & 0 & 0 & 0 & 0 & 20 & $100 \%$ \\
\hline & & & & & & & & & & & & $98.65 \%$ \\
\hline
\end{tabular}

First of all, we notice the high recognition accuracy (98.65\%) reached by the proposed method in spite of the issues cited above. Those issues are respectively, the existence of outlier samples, large variation, in terms of visual features, within some varieties (as shown in Fig. 2(a), Fig. 2(b) and Fig. 2(c)) and the high visual resemblance between some varieties (as shown in Fig. 3(a), Fig. 3(b) and Fig. 3(c)). Indeed, these issues make the recognition task more complicated, as they potentially lead to confuse some varieties with others.

Nonetheless, we see that in 8 from the 11 varieties, the recognition accuracy was $100 \%$. Those varieties are Ajina, Degla Bayda, Dfar lgat, Dgoul, Litima, Tarmount, Tantbucht and Tinisin, respectively. For Ajina variety, this high rate may be due to its big size compared to the other varieties. Nevertheless, reaching an accuracy of $100 \%$ in Tarmount and Tantbucht is, indeed, very encouraging because samples within these varieties are quite similar. In addition, it should be mentioned that the varieties that deeply look alike have achieved high recognition rates. For instance, most samples from Litima and Bayd hmam varieties which cannot, at most, be distinguished even by humans, have successfully recognized (100\% and $95.2 \%$, respectively). Moreover, we notice that only one sample from each of Hamarya and Bouaarous varieties has been wrongly assigned to each other. This slight confusion is totally justified by considering the extreme visual resemblance, in terms of shape and color, of some samples belonging to these latter varieties. Despite the fact that fully mature samples from Tinisin are very similar, in terms of color, to those from Dgoul, our method has successfully recognized both of them. Actually, these high rates prove the distinguishing strength of our method and its ability to deal with such confusing varieties.

To demonstrate that removing outliers boosts the learning process and improve the recognition rate, we have examined the proposed method by excluding the outlier removal module. An accuracy of $94.37 \%$ has been reached compared to $98.65 \%$ which is yielded by removing outliers from training images. 


\subsubsection{Second experiment}

In this experiment, we estimate the processing time needed for recognition. The processing time is amongst the most important criterions to be taken into consideration in assessing recognition systems. This criterion becomes quite crucial for the real-time recognition systems. Table 3 describes the major components of our system and the processing time required by each of them.

Table3: Processing time required by the major components of the proposed system.

\begin{tabular}{|l|l|}
\hline Component & $\begin{array}{l}\text { Processing time (per second } \\
\text { and per variety) }\end{array}$ \\
\hline Features extraction & $10.75(0.17$ per image) \\
\hline Detecting the optimal number of clusters & 0.14 \\
\hline Remove outliers & 0.16 \\
\hline Training (GMM+EM) & 0.73 \\
\hline Recognition & 0.00034 (for 20 test images) \\
\hline
\end{tabular}

The processing time required for training is less important than that of testing because the first is performed offline, while the second is performed online. By summing up the values in Table 3, we find that only $7.83 \mathrm{sec}$ (i.e., $((0.17 \mathrm{x} 40)+0.14+0.16+0.73))$ is needed to train one variety (i.e., $86.13 \mathrm{sec}$ to train the 11 varieties). To recognize a sample, two processes are needed, features extraction which cost 0.17 seconds for one image and recognition, which cost $0.00001 \mathrm{sec}$ (i.e., $0.00034 / 20$ ). Hence, our proposed method would satisfy the exigencies of a real-time recognition system.

\subsubsection{Third experiment}

Although, as mentioned before, the studies concerned with date recognition are very scarce, we devote this experiment to compare the proposed method with three others, which are proposed in [15], [16] and [17]. Table 4 outlines the three methods and their experimental settings.

Table 4: Experimental settings of the related methods.

\begin{tabular}{|c|c|c|c|}
\hline & Method in [15] & Method in [16] & Method in [17] \\
\hline Features & $\begin{array}{l}\text { Weber Local Descriptor } \\
\text { (WLD), Local } \\
\text { Patterns (LBP) }\end{array}$ & $\begin{array}{l}\text { Color mean and standard } \\
\text { deviation (from the } 3 \text { RGB } \\
\text { channels), Area, Perimeter, } \\
\text { Eccentricity, Major and } \\
\text { Minor axis length, Entropy } \\
\text { (from the } 3 \text { RGB } \\
\text { channels)and Energy } \\
\text { measures extracted from the } \\
\text { Grey-Level Co-occurrence } \\
\text { Matrix (GLCM) }\end{array}$ & $\begin{array}{l}\text { Color mean and standard } \\
\text { deviation, for each of the } \\
\text { three RGB channels }\end{array}$ \\
\hline Classifier & $\begin{array}{l}\text { Support } \text { Vector } \\
\text { (SVM) }\end{array}$ & $\begin{array}{l}\text { Artificial Neural Network } \\
\text { (ANN) }\end{array}$ & $\begin{array}{ll}\text { Probabilistic } & \text { Neural } \\
\text { Network (PNN) } & \end{array}$ \\
\hline Size of the used dataset & 200 images & 140 images & 200 images \\
\hline Number of varieties & 4 & 7 & 5 \\
\hline
\end{tabular}

From the table above, we can observe the substantial differences between our experimental settings and those of the mentioned methods. First, the number of images used in each of them is very limited, which is practically insufficient to really investigate the method performance. Second, the 
number of varieties is too little, while, in real life practical applications much more others have to be recognized. Table 5 shows the average recognition accuracy yielded by each method.

Table 5: Average recognition accuracy yielded by each method.

\begin{tabular}{|l|c|c|c|c|c|}
\hline Method & Method in [15] & Method in [16] & Method in [17] & $\begin{array}{c}\text { Ours } \\
\text { (by excluding outlier } \\
\text { removal module) }\end{array}$ & $\begin{array}{c}\text { Ours } \\
\text { (Including outlier } \\
\text { removal module) }\end{array}$ \\
\hline $\begin{array}{l}\text { Average } \\
\text { accuracy } \\
(\%)\end{array}$ & 55.44 & 93.83 & 73.20 & $\mathbf{9 4 . 3 7}$ & $\mathbf{9 8 . 6 5}$ \\
\hline
\end{tabular}

From Table 5, we notice that the proposed method (by including the outlier removal module) has significantly outperformed the methods in [15] and [17] with $43.21 \%$ and $25.45 \%$, respectively. In addition, it exceeds the method in [16] with $4.82 \%$. The noticeable difference in the recognition accuracy between the previous methods and ours can be attributed to several reasons. First of all, we believe that the main reason behind that is the inability of these methods to properly deal with the emerging challenges from the dataset we introduced in this paper. Those methods aren't capable to deal with the confusing varieties as well as the large variation within some varieties. Besides, they don't take into account the presence of outlier samples, which could decrease the recognition accuracy. We observe also that even by excluding the outlier removal module, the proposed method has outperformed the competing methods, which prove its superiority.

To check the above interpretations, we report the accuracy per variety yielded by each method, as shown in Fig. 7. For the method in [16], we note that the lowest accuracies are yielded by Degla bayda, Dfar lgat, Bouaarous, Tarmount and Hamraya varieties. Similarly, in the method in [17], we observe that the lowest accuracies are those yielded by Dfar lgat, Bayd hmam, Tarmount and Litima. Thus, the problem arises with the varieties having roughly the same visual characteristics. Therefore, these methods failed to distinguish between the highly similar varieties, which confirms our interpretations. For the method in [15], the accuracies yielded by most of the varieties are relatively low, which may be because of the lack of color descriptors with the used features.

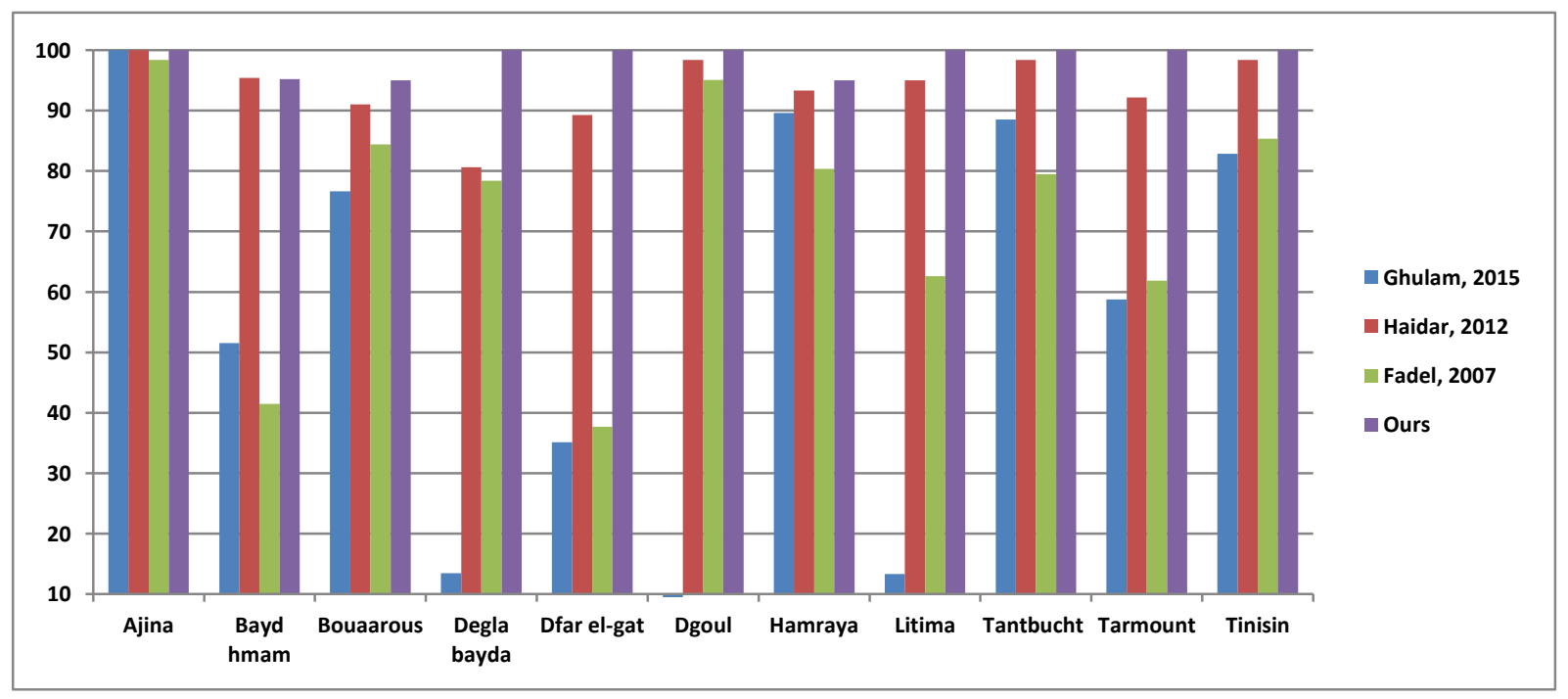

Fig. 7: The recognition accuracy, per variety, yielded by each method. 


\subsubsection{Fourth experiment}

The current experiment aims to examine the recognition strength of each of the features we have used. The accuracy per variety yielded by each feature combination is shown in Fig.8. The first note we can make is that the fusion of all the features has yielded, in most varieties except Hamaraya and Bouaarous, the highest accuracy. The strong visual resemblance between these two varieties makes very difficult to distinguish them from each other. By taking a look on the confusion matrix, we can see that one sample from each of the varieties has mis-classified to each other. Color features have shown a well performance for most of the varieties, which proves the ability of color as decisive features. However, color features have yielded a comparatively low accuracy for Dfar lgat $(78.11 \%)$ because using color alone is not enough to distinguish this variety from the deeply similar varieties like Degla Bayda. For the GLCM, we note that the accuracy scored in most varieties is relatively low. As instance, the accuracy for Bayd hmam was $48.14 \%$, which can be explained by the fact that texture of this latter variety is analogous with that of some others such as Litima. Meanwhile, GLCM has reached an accuracy of $86.45 \%$ in Bouaarous variety.

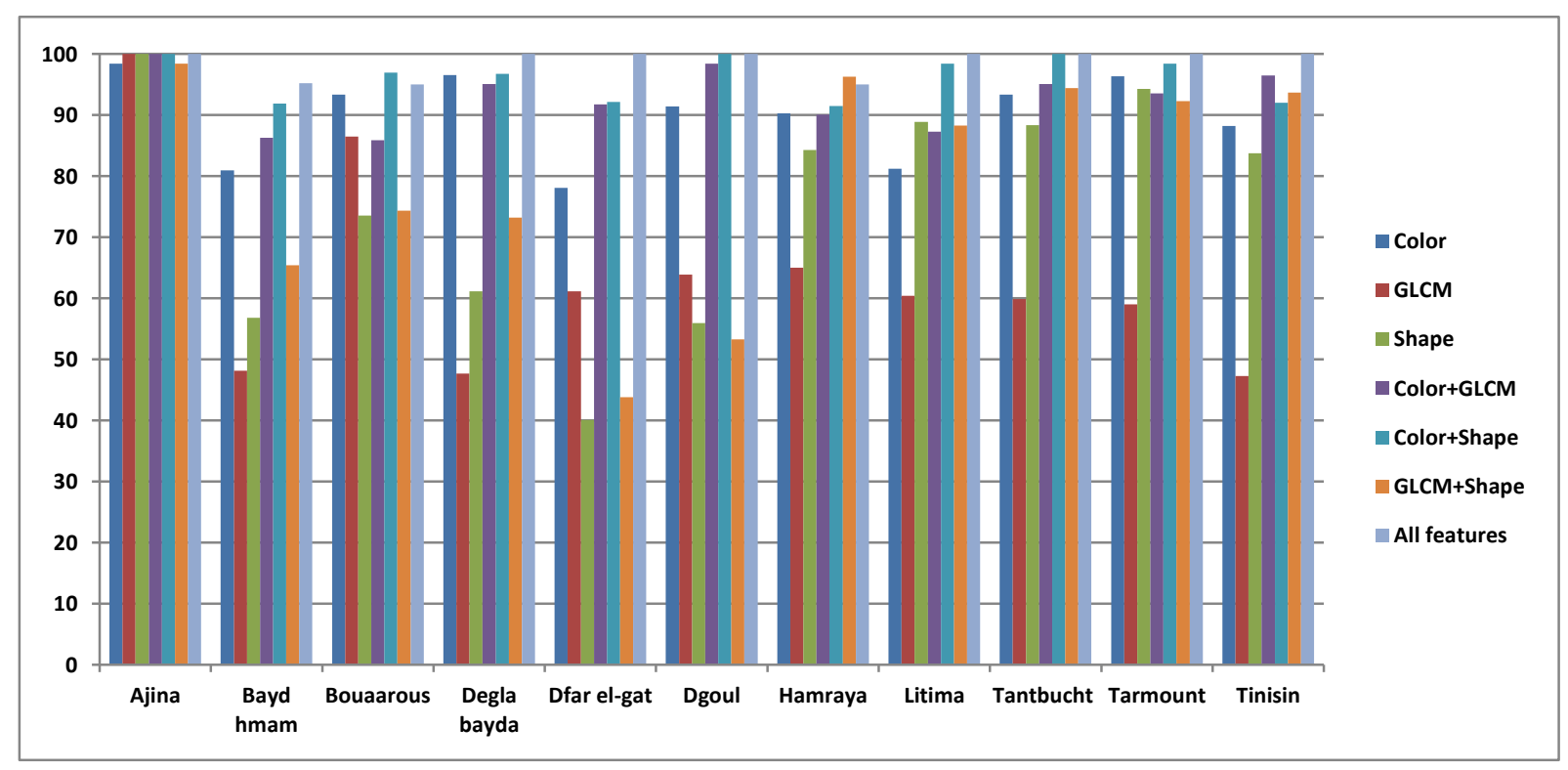

Fig. 8: The recognition accuracy, per variety, yielded by each combination.

Shape features have performed well in some varieties such as Tarmount (94.26\%), but fail in some others such as Dfar lgat (40.08\%), as samples appertaining to this variety could be confused with other samples, from the other varieties, having roughly the same shape and size.

Table 6 shows the average accuracy of each combination. From this table, we observe that the lowest accuracy is that of the GLCM (63.54\%). Meanwhile, color features have demonstrated their importance by reaching an average accuracy of $89.84 \%$. In addition, combination of color with shape has yielded better than the combination of color with the GLCM. By combining all the features we gain a recognition rate of $98.65 \%$.

Table 6: Average recognition accuracy yielded by each combination.

\begin{tabular}{|l|l|l|l|l|l|l|l|}
\hline Combination & Color & GLCM & Shape & $\begin{array}{l}\text { Color+ } \\
\text { GLCM }\end{array}$ & $\begin{array}{l}\text { Color+ } \\
\text { Shape }\end{array}$ & $\begin{array}{l}\text { GLCM+ } \\
\text { Shape }\end{array}$ & $\begin{array}{l}\text { All } \\
\text { features }\end{array}$ \\
\hline $\begin{array}{l}\text { Average } \\
\text { accuracy (\%) }\end{array}$ & 89.84 & 63.54 & 75.18 & 92.71 & 96.19 & 79.41 & 98.65 \\
\hline
\end{tabular}




\subsubsection{Fifth experiment}

The present experiment is carried out to compare the performance of the proposed method against that of the humans in recognizing date fruit varieties. For this end, we have asked 9 different persons to recognize 11 date samples (Fig. 9), where each sample belongs to a particular variety. We have restricted ourselves to 11 samples because it is boring for persons to exhaustively recognize a big number of samples.

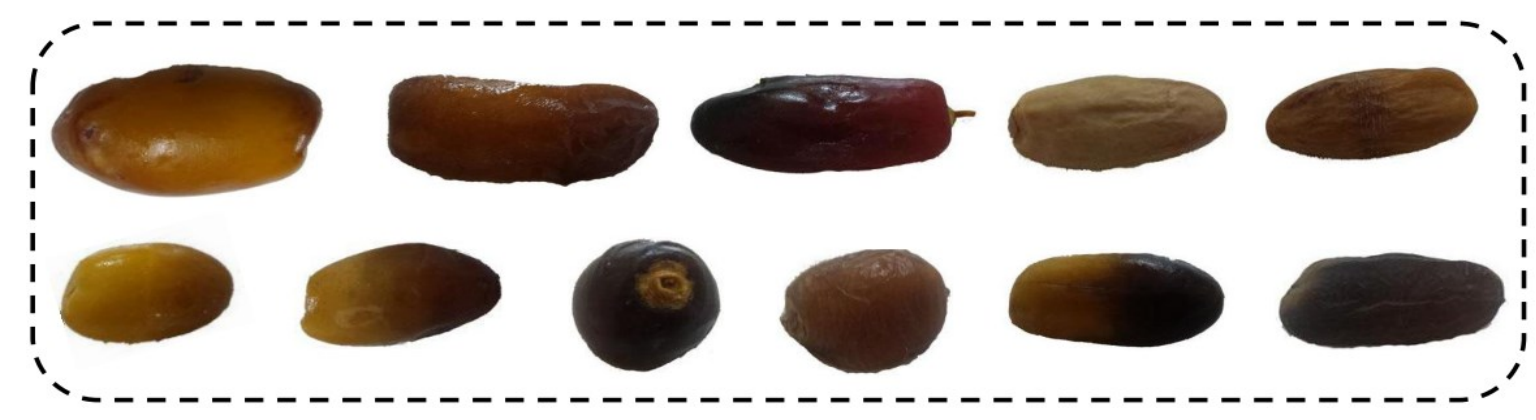

Fig. 9: The 11 samples used for test the human performance.

Fig. 10 shows the accuracy, per variety, yielded by our method compared to human performance. It should be mentioned that the average precision for the human recognition is $91.91 \%$ compared to 98.65\% for the proposed method. From Fig. 10, we can see that the proposed method has outperformed the human performance in certain varieties, which is not the case for certain others. For example, for both Bayd hmam and Litima varieties, the precision yielded by our method was higher than that of humans. This is because, as we have already mentioned, humans confuse those two varieties with each other. This confusion arises because of the strong visual resemblance between the two varieties. At the opposite, we observe that humans have successfully distinguished Degla bayda from Dfar el-gat in spite of the strong visual resemblance between the two varieties. For some other varieties such as Tinisin, we observe that both humans and the proposed method have successfully recognized test samples.

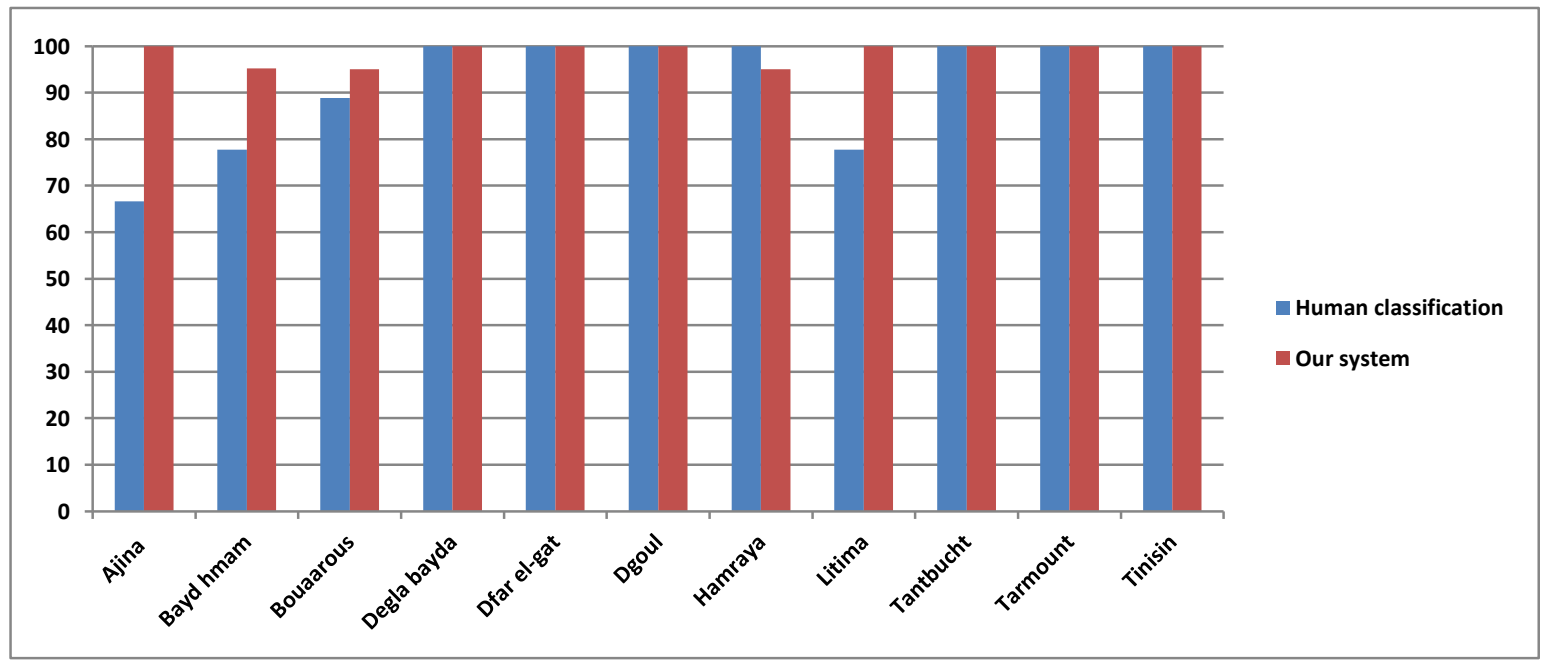

Fig. 10: The recognition accuracy, per variety, yielded by our method compared to human performance 


\section{Conclusion}

In this paper, we have proposed a method for automatic date fruit recognition. Specifically, we prune training samples of each variety from outlier samples which could negatively affect the recognition results. For this purpose, we have used the Pruning Local Distance-based Outlier Factor (PLDOF) method. Samples appertaining to the same variety may significantly differ in terms of visual characteristics. In order to take this intra-variation into account, we have represented each variety with Gaussian Mixture Model, where each component corresponds to one visual appearance. Since the number of components per GMM greatly affects the recognition accuracy, Davies-Bouldin index has been adopted to estimate it with precision. Our proposed method makes several noteworthy contributions to the literature. First, it is capable to recognize samples in different maturity stages and to distinguish between the deeply confusing varieties. Second, it is able to alleviate the negative effect of outlier samples. Third, it is fully automatic because neither human involvement nor physical measurements are needed. Fourth, it is computationally fast, as it has been shown in the experiments. To investigate the performance of the proposed method, we have introduced a new date benchmark that made up of 660 samples from 11 varieties. Experimental results prove the capability of the proposed method in recognizing date samples in spite of the challenges mentioned above. In addition, experimental results showed that our method has significantly outperformed several methods from the state of the art. In a future work, one can investigate the performance of our method in date grading i.e., classifying samples belonging to the same variety into different grades. Another idea to be investigated is the use of other image acquisition instruments e.g., infrared and ultraviolet in order to capture information outside of the visible range. 


\section{References}

[1] A. Amanat, M. Waly, M. Mohamed Essa, S. Devarajan, "Nutritional and medicinal value of date fruit". A. Manickavasagan et al. (Eds.). Dates: Production, Processing, Food, and Medicinal Values. CRC Press, 361-376, 2012.

[2] M. Al-Farsi, C. Alasalvar, A. Morris, M. Baron, F. Shahidi, "Compositional and sensory characteristics of three native sun-dried date (Phoenix dactylifera L.) varieties grown in Oman". Journal of Agriculture and Food Chemistry, 53(19), 7586-7591, 2005.

[3] Y. Mimouni, O. Siboukeur, "Etude des propriétés nutritives et diététiques de sirops de dattes extraites par diffusion en comparaison avec des sirops à haute teneur en fructose (isoglucose) issus de l'industrie de l'amidon". Annales des Science et Technologie, 3, 1-11, 2011.

[4] N. Bouguedoura, M. Bennaceur, S. Babahani, S.E. Benziouche, "Date palm status and perspective in Algeria". Al-khayri, J.M. et al. (Eds.). Date Palm Genetic Resources and Utilization. Springer, 125-168, 2015.

[5] S. Besbes, L. Drira, C. Blecker, C. Deroanne, H. Attia, "Adding value to hard date (Phoenix dactylifera L.): Compositional, functional and sensory characteristics of date jam". Food Chemistry, 112, 406-411, 2009.

[6] H.A. Khatchadourian, W.N. Sawaya, M. Ayaz, M.M. Al-Mohemmed, "Processing date varieties into pickles". International Journal of Food Science and Technology, 22, 243-247, 1987.

[7] S.M. Al-Orf, M.H.M. Ahmed, N. Al- Atwai, H. AlZaidi, A. Dehwah, S. Dehwah, "Review: Nutritional Properties and Benefits of the Date Fruits (Phoenix dactylifera L.)". Bulletin of the National Nutrition Institute of the Arab Republic of Egypt, 39, 98-129, 2012.

[8] S. Al-Hooti, J.S. Sidhu, H. Qabazard, "Physicochemical characteristics of five date fruit cultivars grown in the United Arab Emirates". Plant Foods Human Nutrition, 50(2), 101-113, 1997.

[9] B. Lambiote, "Some aspects of the role of dates in human nutrition". In: the First International Symposium on Date Palm, 573-579, 1982.

[10] Y. Mimouni, O. Siboukeur, Z. Bayoucef, "Post-prandial blood glucose of four cultivars of date palm (Phoenix dactylifera) in healthy subjects". Revue ElWahat pour les Recherches et les Etudes, 8(1), 62-72, 2015.

[11] A. Manickavasagan, N.K. Al-Mezeini, H.N. Al-Shekaili, "RGB color imaging technique for grading of dates". Scientia Horticultuare, 75, 87-94, 2014.

[12] Abdellahhalimi, A. Roukhe, B. Abdenabi, N. El Barbri, "Sorting dates fruit bunches based on their maturity using camera sensor system". Journal of Theoretical and Applied Information Technology, 56(2): 325-337, 2013.

[13] S. Al-Rahbi, A. Manickavasagan, R. Al-Yahyai, L. Khriji, P. Alahakoon, "Detecting surface cracks on dates using color imaging technique". Food Science and Technology Research, 19(5), 795-804, 2013.

[14] Y.I. Al-Ohali, "Computer vision based date fruit grading system: design and implementation". Journal of King Saud University - Computer and Information Science, 23(1), 29-36, 2011.

[15] M. Ghulam, "Date fruits classification using texture descriptors and shape-size Features". Engineering Applications of Artificial Intelligence, 37, 361-367, 2015.

[16] A. Haidar, D. Haiwei, N. Mavridis, "Image-based date fruit classification". In: the 4th International Congress on Ultra-Modern Telecommunications and Control Systems and Workshops (ICUMT), 357-363, 2012.

[17] M. Fedal, "Date fruits classification using probabilistic neural networks". Agricultural Engineering International : the CIGR Ejournal, 4, 1-6, 2007.

[18] D.J. Lee, K. James, X. Guangming, "Rapid color grading for fruit quality evaluation using direct color mapping". IEEE Transactions an Automation Science and Engineering, 8(2), 292-303, 2010 . 
[19] K.M. Ismail, K.A. Al-Gaadi, "Development of an electronic sensor for date sorting based on moisture content". Misr Journal of Agricultural Engineering, 26(4), 1923-1932, 2009.

[20] R. Pourdarbani, H.R. Ghassemzadeh, H. Seyedarabi, F.Z. Nahandi, M.M. Vahed, "Study on an automatic sorting system for Date fruits", Journal of the Saudi Society of Agricultural Science, 14(1), 83-90, 2015.

[21] S. Al-Rahbi, A. Manickavasagan, G. Thomas, "Back propagation neural network (BPNN) to detect surface crack on dates using RGB images". Journal of Medical and Bioengineering, 4(1), 67-70, 2015.

[22] A.A. Al-Janobi, "Date inspection by color machine vision". Journal of King Saud University. Agricultural Sciences, 12(1), 69-79, 2000.

[23] S.H. Mohana, C.J. Prabhakar, "A novel technique for grading of dates using shape and texture features". Machine Learning and Applications: An International Journal, 1(2), 15-29, 2014.

[24] A.I. Hobani, A.N. Thottam, K.A.M. Ahmed, "Development of a neural network classifier for Date fruit varieties using some physical attributes". King Saudi University - Agricultural Research Center 126, 5-18, 2003.

[25] R. Pamula, J.K. Deka, S. Nandi, " An Outlier Detection Method based on Clustering”. In the Second International Conference on Emerging Applications of Information Technology, 253-256, 2011.

[26] K.V. Mardia, "Measures of multivariate skewness and kurtosis with applications". Biometrika, 57(3), 519-530, 1970.

[27] N. Armanfard, J.P. Reilly, M. Komeili, "Local Feature Selection for Data Classification". IEEE Transactions on Pattern Analysis and Machine Intelligence, 38(6), 1217-1227, 2016.

[28] D. Zhang, M.M. Islam, G. Lu, “A review on automatic image annotation techniques". Pattern Recognition, 45, 346-362, 2012.

[29] R.M. Haralick, K. Shanmugam, I.H. Dinstein, "Textural features for image classification". IEEE Transactions on Systems, Man and Cybernetics, 6, 610-621, 1973.

[30] D. L. Davies, D. W. Bouldin, "A Cluster Separation Measure". IEEE Transactions on Pattern Analysis and Machine Intelligence, 1(2), 224-227, 1979.

[31] J. Yang, K. Yu, Y. Gong, T. Huang, "Linear spatial pyramid matching using sparse coding for image classification". IEEE Conference on Computer Vision and Pattern Recognition, 2009.

[32] O. Boiman, E. Shechtman, M. Irani, "In defense of nearest-neighbor based image classification". IEEE Conference on Computer Vision and Pattern Recognition, 2008.

[33] A. Bosch, A. Zisserman, X. Munoz, "Image classification using random forests and ferns". IEEE 11th International Conference on Computer Vision, 2007.

[34] B. Harish, A. Hedge, O. Venkatesh, D. Spoorthy, D. Sushma, "Classification of plant leaves using Morphological features and Zernike moments". IEEE International Conference on Advances in Computing, Communications and Informatics, 2013.

[35] E. Elhariri, N. El-Bendary, A. E. Hassanien, "Plant classification system based on leaf features". IEEE 9th International Conference on Computer Engineering \& Systems, 2014.

[36] M. E. Nilsback, A. Zisserman, "Automated flower classification over a large number of classes". IEEE Sixth Indian Conference on Computer Vision, Graphics \& Image Processing, 2008.

[37] H. Farsi, H. K. Alaei, "A Novel Algorithm to Tackle Eyeglasses and Beard Issues in Facial IR Recognition”. Electronic Letters on Computer Vision and Image Analysis, 14(1), 2015.

[38] A. Kacem, A. Khémini, A. Belaid, "A PGM-based System for Arabic Handwritten Recognition”. Electronic Letters on Computer Vision and Image Analysis, 13(3), 2014.

[39] V. Berthiaume, M. Cheriet "Handwritten Digit by Fourier-Packet Descriptors". Electronic Letters on Computer Vision and Image Analysis, 11(1), 2012.

[40] M. J. Choi, A. Torralba, A. S. Willsky, "A Tree-Based Context Model for Object Recognition". 
IEEE Transactions on Pattern Analysis and Machine Intelligence, 34(2), 2012.

[41] B. S. Bennedsen, D. L. Peterson A. Tabb, "Identifying Apple Surface Defects using Principal Components Analysis and Artificial Neural Networks". Transactions of American Society of Agricultural and Biological Engineers, 50(6), 2007.

[42] D.G. Kim, T.F. Burks, J. Qin, D.M. Bulanon, “Classification of Grapefruit Peel Diseases using Color Texture Feature Analysis". International Journal of Agricultural and Biological Engineering, 2(3), 2009.

[43] A. Esehaghbeygi, M. Ardforoushan, S.A.H. Monajemi, A.A. Masoumi, "Digital Image Processing for Quality Ranking of Saffron Peach”. International Agrophysics, 24(2), 2010. 